\title{
Performance analysis of wavelength division- and subcarrier-multiplexing (WDM-SCM) transmission using fibre Brillouin amplification
}

Y.-H. Lee

J. Wu

M.-S. Kao

H.-W. Tsao

\begin{abstract}
A subcarrier multiplexing based optical wavelength division multiplexing system with fibre Brillouin amplification (FBA) is analysed. In the optical domain, a pump laser is tuned to amplify the corresponding optical carrier by FBA for the desired group of SCM signals. In the electrical domain, a microwave tuner is used to select the desired channel in the selected SCM group. This system has the benefits of eliminating the need for polarisation control, the ability to cancel phase noise due to the 'squaring' photodetection process of the selected optical carrier together with its SCM channels, and enhancement of optical receiver sensitivity by amplification of the carrier. Comparisons with other systems are also presented.
\end{abstract}

\section{Introduction}

Efficient multichannel transmission can be achieved with an optical carrier using a subcarrier multiplexing (SCM) technique $[1,2]$ which provides an attractive feature for exploiting the wide bandwidth of single-mode fibres with commercially available microwave electronic components. Recently, some systems that combine wavelength division multiplexing (WDM) and SCM to further increase transmission capacity have been reported $[3,4]$. These systems use an optical wavelength filter to select the desired group of SCM signals which is transceived with the intensity modulation/direction detection (IM/DD) technique. However, the additional loss penalty introduced by the wavelength filter restricts the system operating range.

In this paper we propose a WDM-SCM system as shown in Fig. 1 where channel selection consisted of two stages. The first stage selection in WDM (optical tuning) is based on the selective amplification of the chosen optical carrier by employing fibre Brillouin amplification (FBA) $[5,6]$ prior to photodetection. This is possible because the residual optical carrier in the spectra of SCM

Paper 8782J (E13), first received 19th August 1991 and in revised form 14th January 1992

Y.-H. Lee, J. Wu and H.-W. Tsao are with the Department of Electrical Engineering, National Taiwan University, Taipei, Taiwan, Republic of China

M.-S. Kao is with the Department of Communication Engineering, National Chiao Tung University, Hsincha, Taiwan, Republic of China. signals [7] can be easily amplified by narrowband Brillouin amplification with a tunable pump laser. The second stage selection in SCM (microwave tuning) is accomplished by tuning the microwave voltage control oscillator (VCO) to cause the desired SCM channel falling into the passband of a bandpass filter (BPF), then it can be demodulated to obtain the baseband signal.

In this system, we use an extremely narrowband optical amplifier to amplify the residual optical carrier of the SCM system, which is achieved elegantly within the transmission fibre by backward stimulated Brillouin scattering (SBS). This process has a bandwidth of $15-25 \mathrm{MHz}$ for $\mathrm{CW}$ pump and it can be broadened by frequency modulating the pump laser $[8,9]$. To obtain Brillouin amplification, it is necessary to have a pump laser at the receiver to feed optical energy back along the fibre towards the transmitter. Significant gains can be achieved at milliwatt-pumping power levels in a long fibre [10].

This system has some advantages. First, the need of polarisation control can be eliminated and phase noise can be reduced [11]. Because the polarisation state and phase noise of SCM channels and their corresponding optical carrier are nearly the same; after the squaring photodetection process, the cross terms of the optical carrier and SCM channels thus produce microwave signals without the deterioration due to polarisation mismatch and phase noise [8]. Secondly, the preamplification of a modulated optical signal by an extremely narrowband amplifier enhances the signal-tonoise ratio [11]. Thirdly, the wavelength selection is accomplished by the FBA together with its optical amplification without the insertion loss caused by the optical wavelength filter.

\section{Analysis}

\subsection{System description}

Consider a WDM-SCM system as shown in Fig. 1. At the transmitter, we modulate $N_{C}$ VCO outputs by corresponding baseband signals (called 'channels') and combine these signals to produce the composite microwave signal. The baseband signals may be either analogue or digital. Then we use these signals to modulate the corresponding $N_{W}$ lasers by $N_{W}$ external amplitude modulators, respectively. The $N_{W}$ optical carriers, each carrying their corresponding $N_{C}$ subcarriers, are then multiplexed to form the WDM-SCM signal which includes $N_{W} N_{C}$ channels.

IEE PROCEEDINGS-J, Vol. 139, No. 4, AUGUST 1992 
The spectra of the subcarriers expand symmetrically around their corresponding wavelength $\hat{\lambda}_{i}$ as shown in Fig. 2; these $2 N_{C}$ sidebands come from the $N_{C}$ channels. where $\Delta v_{B}$ and $\Delta v_{P}$ are the full widths at half maximum (FWHM) of the Brillouin-gain profile and pump laser, respectively; $v_{B}$ is the Brillouin frequency shift with a cor-
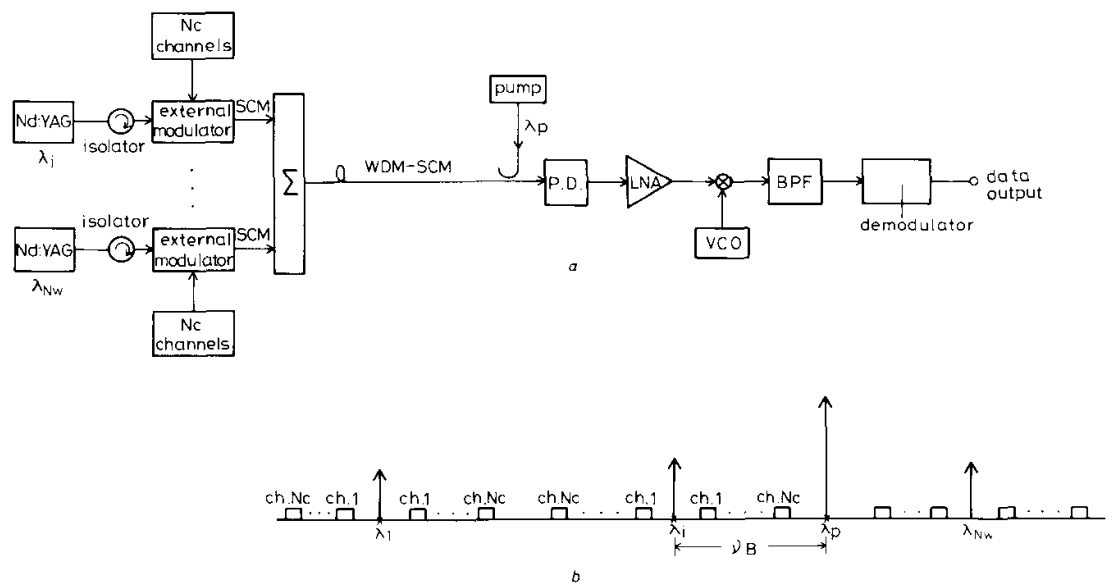

Fig. $1 W D M-S C M$ system

$a$ block diagram $\quad b$ optical spectrum

At the receiver, we tune the frequency of the pump laser to amplify the desired optical pilot carrier by FBA. This process generates noises including the amplified spontaneous emission noise (ASEN) $[6,12]$. The optical signal and noises are then converted by the photodetector into a photocurrent. The noises include the spontaneous emission, the thermal noise, the shot noise, the intermodulation distortion (IMD) [1], the beat noises of unselected optical carriers and their own SCM channels, the signal-spontaneous beat noises, and the beat noises between any cross-wavelength terms (see Fig. 3).

\subsection{Fibre brillouin amplification and amplified} spontaneous emission noise (ASEN)

We assume that the Brillouin gain has a Lorentzian spectral profile given by [15]

$$
g(v)=\left[\frac{\Delta v_{B}^{2} / 4}{\left(v-v_{B}\right)^{2}+\Delta v_{B}^{2} / 4}\right]\left(\frac{\Delta v_{B}}{\Delta v_{B}+\Delta v_{P}}\right) g_{0}
$$

responding peak gain $g_{0}$ without considering the effect of $\Delta v_{P}$.

For $\Delta v_{B} \gg \Delta v_{S}\left(\Delta v_{S}\right.$ is the FWHM of the Stoke wave), we may consider all the Stoke waves amplified by the SBS gain $G$, which can be expressed by [15] (see Fig. 4)

$$
G=\exp \left[\frac{g\left(v_{B}\right) P}{2 \alpha A_{e}}\right]
$$

where $P$ is the pump power, $\alpha$ is the absorption coefficient $\left(m^{-1}\right), A_{e}$ is the effective core area of the fibre and the factor 2 accounts for the random polarisation of the waves.

The noise in FBA results from the spontaneous Brillouin scattering. In the undepleted pump condition, the ASEN can be calculated exactly, in terms of spontaneous photon per mode, for long fibre length, as given by [ 6 , 12]

$$
N_{s}(v)=\frac{M}{\Gamma}\left(e^{\Gamma}-1\right)(\Gamma+1)
$$

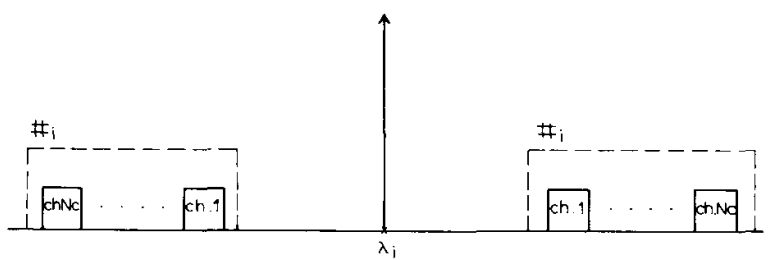

Fig. 2 Spectrum of the ith optical carrier together with its $N_{C} S C M$ channels

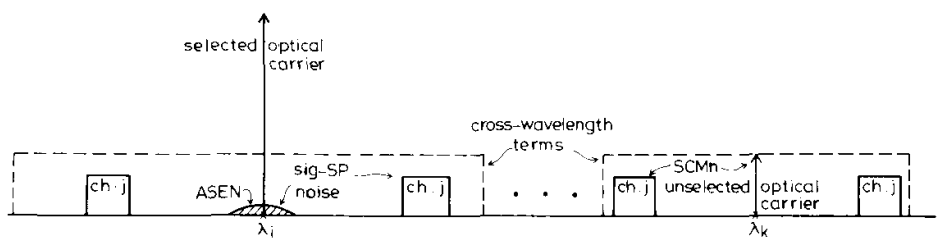

Fig. 3 Noise spectra in WDM-CSCM system 
with $M$ and $\Gamma$ defined as

$$
M=\frac{1}{1-e^{-h v_{B} / k T}}
$$

and

$$
\Gamma=\frac{g(v) P}{\alpha A_{e}}
$$

where $h v_{B}$ is the Stoke shift energy, $k$ is the Boltzmann constant and $T$ is the absolute temperature.

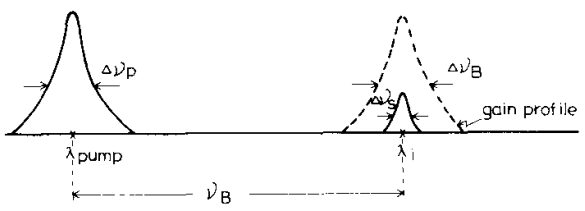

Fig. 4 Optical spectrum of the selected $k t h$ wavelength and pump laser together with gain profile

Then we may obtain the total ASEN power $P_{s p}$ and its electric field as [12]

$$
\begin{aligned}
& P_{s p}=\int_{0}^{\infty} N_{s}(v) h v d v \\
& E_{s p}(t)=\sqrt{ }\left(P_{s p}\right) \cos \left(\omega_{\lambda_{i}} t+\Phi_{s p}\right)
\end{aligned}
$$

where $\omega_{\lambda_{i}}$ and $\Phi_{s p}$ are the central frequency and the random phase of the ASEN. Because of random polarisation, the effective power is only $P_{s p} / 2$.

\subsection{Selection in WDM}

If we want to choose the ith optical carrier with frequency $f_{\lambda_{i}}$ (the $i$ th wavelength) in the WDM, we have to tune the pump laser frequency, $f_{\text {pump }}$, to amplify the desired optical carrier as

$$
f_{\text {pump }}=f_{\lambda_{i}}+v_{B}
$$

Then the photocurrent at the output of the photodetector, $i(t)$, can be expressed as

$$
i(t)=R\left|E_{o}+E_{S}+\overline{E_{o}}+\overline{E_{S}}+E_{s p}\right|^{2}
$$

where $R(A / W)$ is the responsivity of the photodetector. The last term in the above equation is given in eqn. 7 . The first four terms which represent, respectively, the electric field of the selected $i$ th optical carrier amplified by FBA $\left(E_{o}\right)$, the electric fields of the selected SCM signals in the ith carrier $\left(E_{s}\right)$, the electric fields of the total unselected optical carriers $\left(\overline{E_{o}}\right)$, and the electric fields of the total unselected SCM channel $\left(\overline{E_{S}}\right)$, are given as

$$
\begin{aligned}
E_{\mathrm{o}}(t)= & \sqrt{ }\left(2 G P_{S}\right) \cos \left(\omega_{\lambda_{i}} t+\Phi_{\lambda_{i}}\right) \\
E_{S}(t)= & \sum_{l=1}^{N_{C}} m_{l, i} \sqrt{ }\left(P_{S} / 2\right)\left\{\cos \left[\left(\omega_{\lambda_{i}}-\omega_{l}\right) t+\Phi_{\lambda_{i}}\right]\right. \\
& \left.+\cos \left[\left(\omega_{\lambda_{i}}+\omega_{l}\right) t+\Phi_{\lambda_{i}}\right]\right\} \\
\overline{E_{o}(t)}= & \sum_{k=1, k \neq i}^{N_{W}} \sqrt{ }\left(2 P_{S}\right) \cos \left(\omega_{\lambda_{k}} t+\Phi_{\lambda_{k}}\right) \\
\overline{E_{S}(t)=} & \sum_{k=1, k \neq i}^{N_{W}} \sum_{l=1}^{N_{C}} m_{l, k} \sqrt{ }\left(P_{S} / 2\right)\left\{\cos \left[\left(\omega_{\lambda_{k}}-\omega_{l}\right) t+\Phi_{\lambda_{k}}\right]\right. \\
& \left.+\cos \left[\left(\omega_{\lambda_{k}}+\omega_{l}\right) t+\Phi_{\lambda_{k}}\right]\right\}
\end{aligned}
$$

where $P_{S}$ is the received power of each optical carrier without amplification (assumed equal for all lasers), $G$ is the gain of SBS, $\omega_{1}$ is the microwave subcarrier frequency, $m_{t, k}$ is the modulation index of the $l$ th channel in the $k$ th optical carrier, $\omega_{\lambda_{i}}$ and $\Phi_{\lambda_{i}}$ are the frequency and phase noise of the selected $i$ th optical carrier, and $\omega_{\lambda_{k}}$ and $\Phi_{\lambda_{k}}$ are the frequency and phase noise of the unselected $k$ th optical carrier. If optical modulation depth per channel is the same

$$
m_{l, k}=m
$$

then the total effective optical modulation depth per laser, $m_{o}$, can be expressed as

$$
m_{o}=m \sqrt{ }\left(N_{C}\right)
$$

By substituting eqns. 14 and 15 into eqns. 11 and 13 , we obtain

$$
\begin{aligned}
E_{S}(t)= & \sum_{i=1}^{N_{C}} m_{o} \sqrt{\left(\frac{P_{s}}{2 N_{C}}\right)\left\{\cos \left[\left(\omega_{\lambda_{i}}-\omega_{l}\right) t+\Phi_{\lambda_{i}}\right]\right.} \\
& \left.+\cos \left[\left(\omega_{\lambda_{i}}+\omega_{l}\right) t+\Phi_{\lambda_{i}}\right]\right\} \\
\overline{E_{S}(t)}= & \sum_{k=1, k \neq i}^{N_{W}} \sum_{i=1}^{N_{C}} m_{o} \sqrt{\left(\frac{P_{S}}{2 N_{c}}\right)\left\{\cos \left[\left(\omega_{\lambda_{k}}-\omega_{l}\right) t+\Phi_{\lambda_{k}}\right]\right.} \\
& \left.+\cos \left[\left(\omega_{\lambda_{k}}+\omega_{l}\right) t+\Phi_{\lambda_{k}}\right]\right\}
\end{aligned}
$$

Because each optical carrier $\lambda_{i}$ in WDM is separated widely enough and the frequency response of the photodetector is relatively slow compared with the frequencies of cross-wavelength terms (the cross terms arising from different optical carriers are $2 R E_{\mathrm{o}} \overline{E_{o}}, 2 R E_{\mathrm{o}} \overline{E_{S}}, 2 R E_{\mathrm{o}} E_{\mathrm{S}}$ $2 R \overline{E_{o}} E_{s p}, 2 R E_{S} \overline{E_{S}}$ and $2 R \overline{E_{S}} E_{s p}$ in eqn. 9), we may write the photocurrent as

$$
\begin{aligned}
i(t)= & R\left(E_{o}^{2}+\overline{E_{o}^{2}}+E_{S}^{2}+\overline{E_{S}^{2}}+E_{s p}^{2}+2 E_{o} E_{S}\right. \\
& \left.+2 E_{o} E_{s p}+2 \overline{E_{o} E_{S}}+2 E_{S} E_{s p}\right)
\end{aligned}
$$

\subsection{Selection in SCM}

When we want to choose the $j$ th SCM channel in the $i$ th WDM group, we may tune the frequency of the microwave VCO, $f_{V C o}$, to cause the desired $j$ th channel to fall into the passband of BPF with centre frequency $f_{C}\left(\mid f_{j}\right.$ $-f_{V C O} \mid=f_{C}$ ) and bandwidth $B$ (assuming that the VCO mixer only changes the signal frequency). Then we may obtain the bandpassed signal current as

$$
\begin{aligned}
i_{s i g_{\mathrm{B}}}(t) & =B P F\left\{2 R E_{\mathrm{o}} E_{\mathrm{S}}\left[2 \cos \left(\omega_{V \operatorname{co}} t\right)\right]\right\} \\
& =2 R m_{o} P_{S} \sqrt{\left(\frac{G}{N_{C}}\right) \cos \left(\omega_{C} t\right)}
\end{aligned}
$$

where $B P F[\cdot]$ represents the operation of bandpass filtering. We see that the phase noise in eqn. 19 has been cancelled. The bandpassed noises in eqn. 18 can be expressed, together with their variances, as

$$
\begin{aligned}
& \begin{aligned}
i_{S C M n}(t)= & B P F\left\{2 R \overline{E_{o} E_{S}}\left[2 \cos \left(\omega_{V C o} t\right)\right]\right\} \\
= & 2\left(N_{W}-1\right) R m_{o} P_{S} \cos \left(\omega_{C} t\right) / \sqrt{ }\left(N_{C}\right)
\end{aligned} \\
& \begin{aligned}
\operatorname{var}\left(i_{S C M n}\right)= & 2\left(N_{W}-1\right) R^{2} m_{o}^{2} P_{S}^{2} / N_{C} \\
i_{s i g-s p}(t)= & B P F\left\{2 R E_{S} E_{s p}\left[2 \cos \left(\omega_{V C o} t\right)\right]\right\} \\
= & R m_{o} \sqrt{ }\left(2 P_{S} P_{s p} / N_{C}\right) \\
& \times \cos \left(\omega_{C} t+\Phi_{\lambda_{i}}-\Phi_{s p}\right)
\end{aligned} \\
& \operatorname{var}\left(i_{s i g-s p}\right)=R^{2} m_{o}^{2} P_{S} P_{s p} / N_{C} \\
& \operatorname{var}\left(i_{s h}\right)= 2 e R B P_{S}\left(G+N_{W}-1+N_{W} m_{o}^{2} / 2\right) \\
& \operatorname{var}\left(i_{t h}\right)= 4 N F k T B / r
\end{aligned}
$$


where $e, N F$ and $r$ are the electron charge, the amplifier noise figure $(6 \mathrm{~dB})$, and the load resistance. $i_{S C M_{n}}$ represents the interference from the unselected optical carriers mixed with their own SCM channels. $i_{s i g-s p}$ is the signal-spontaneous noise. The DC power in eqn. 18 [that is, DC terms in $\left.R\left(E_{o}^{2}+\overline{E_{a}^{2}}+E_{S}^{2}+\overline{E_{S}^{2}}\right)\right]$ results in the shot noise, $i_{s h}$. The spontaneous-spontaneous noise (i.e. $R E_{s p}^{2}$ in eqn. 18 ) is relatively small, and the beat noise of the selected optical carrier and the spontaneous noise (i.e. $2 R E_{0} E_{s p}$ ) are not located in the passband of the BPF under the condition that the frequency stability of the pump source is precisely controlled. When the pump source is not located exactly as given in eqn. 8 and there is some random frequency variation, the beat noise of the selected optical carrier and the spontaneous noise may have to be considered in terms of the random frequency variation of the pump sources. In such a case, the Brillouin gain may also change owing to this random frequency variation of the pump laser.

\section{Derivation of CNR}

We shall assume that either the external modulator is perfectly linear $[13,14]$ or the predistortion linearisation technique is used to make the intermodulation distortion less than $-60 \mathrm{dBc}$ [14]. Furthermore if we take the frequency allocation to be single-octave, then the intermodulation distortion will be small and can be ignored

Taking the channels in WDM-SCM to be uncorrelated with each other, we may obtain the CNR at the output of the BPF from eqns. $19-25$ as

$$
\begin{aligned}
C N R= & \frac{2 R^{2} m_{o}^{2} P_{S}^{2} G / N_{C}}{\operatorname{var}\left(i_{S C M n}\right)+\operatorname{var}\left(i_{s i g-s p}\right)+\operatorname{var}\left(i_{s h}\right)+\operatorname{var}\left(i_{i h}\right)} \\
= & \frac{R^{2} m_{o}^{2} P_{S}^{2} G / N_{C}}{R^{2} m_{o}^{2} P_{S} / N_{C}\left[\left(N_{W}-1\right) P_{S}+P_{s p} / 2\right]} \\
& \quad+e R B P_{S}\left(G+N_{W}-1+N_{W} m_{o}^{2} / 2\right)+2 N F k T B / r
\end{aligned}
$$

\section{Examples}

The parameters of optical fibre are given by $A_{e}=47 \mu \mathrm{m}^{2}$, $\alpha=9.5 \times 10^{-5} m^{-1}(0.41 \mathrm{~dB} / \mathrm{km}), \quad g_{0}=4.6 \times 10^{-11}$ $\mathrm{m} / \mathrm{W}, v_{B}=13 \mathrm{GHz}$, and $\Delta v_{B}=22 \mathrm{MHz}$ at $1.32 \mu \mathrm{m}$ wavelength $[10]$. For a system with $R=1[\mathrm{~A} / \mathrm{W}]$, B $=100 \mathrm{MHz}, N_{C}=20$, and $N_{W}=5$, we can obtain the CNR against the pump power as shown in Fig. 5 for

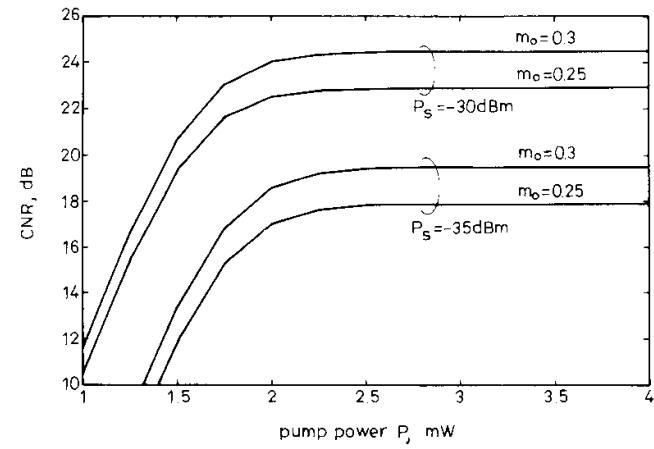

Fig. $5 C N R$ against pump power $P$ for $m_{0}=0.3,0.25$ and $P_{S}=-35$, $-30 \mathrm{dBm}$

$B=100 \mathrm{MHz}$

$N_{w}=5$
$N_{C}=20$

IEE PROCEEDINGS-J, Vol. 139, No. 4, AUGUST 1992 $m_{o}=0.25,0.3$ and $P_{S}=-35,-30 \mathrm{dBm}$, respectively. The CNR increases as the received power, the total optical modulation depth and the pump power increase. The CNR value saturates at a pump power of $2.25 \mathrm{~mW}$ which is determined by the shot noise and the thermal noise when these two noises are of the same order as shown in Fig. 6. Taking $m_{o}=0.25$ and $P_{S}=-35 \mathrm{dBm}$,

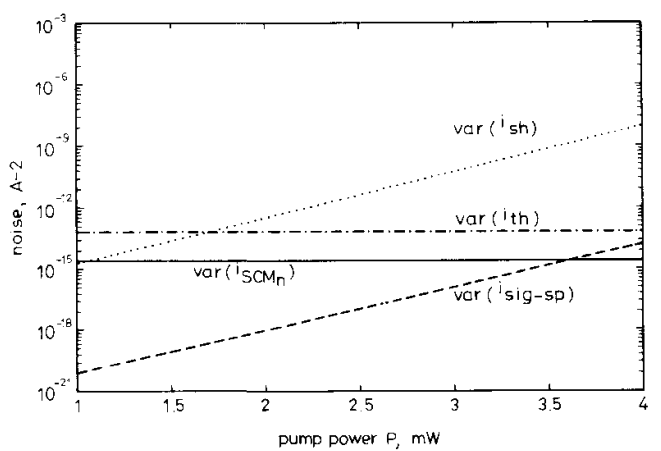

Fig. 6 Bandpass noise currents against pump power for $m_{0}=0.25$ and $P_{S}=-35 \mathrm{dBm}$

we can plot the various noise powers at the output of the BPF against pump power as shown in Fig. 6. In the lower pump power region, the thermal noise, the interference from the unselected optical carriers mixed with their own SCM channels and the shot noise are dominant; whereas in the higher pump power region, the shot noise is dominant and the other noises can be ignored. We also plot the CNR against the channel number of the SCM, $N_{C}$, in terms of the number of the optical carriers in the WDM, $N_{W}$, for $P_{p u m p}=2.5 \mathrm{~mW}, P_{S}=-25 \mathrm{dBm}$ and $m_{o}=0.35$ as shown in Fig. 7. The CNRs increase as $N_{C}$ and $N_{W}$ decrease. We also see that as $N_{C}$ changes from 10 to 50 , the CNR varies approximately from 34 to $27 \mathrm{~dB}$; but when $N_{W}$ changes from 1 to 10 , the CNR remains almost unchanged. Because the optical modulation depth per SCM channel decreases linearly with the number of channels, which in turn reduces the CNR, whereas the optical modulation depth is not affected at all by the number of wavelengths even though receiver noise increases with this number, this effect is minimal.

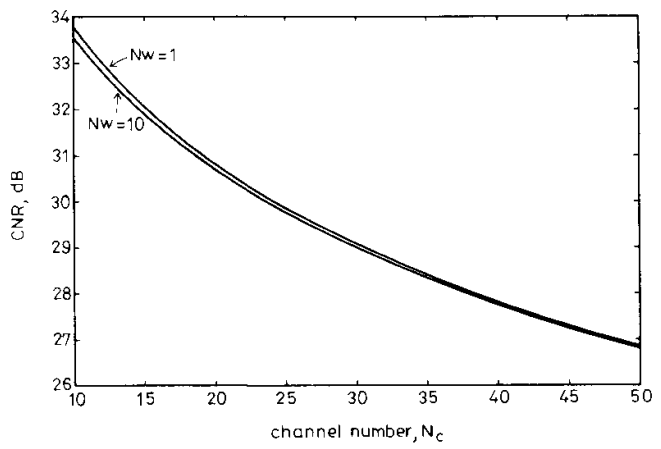

Fig. 7 CNR against channel number of the CSCM, N , for various numbers of optical carriers in the WDM

$N_{\mu}$ for $P_{p u m p}=2.5 \mathrm{~mW}$

$m_{\mathrm{c}}=0.35$

$P_{S}=-25 \mathrm{dBm}$ 
This system suffers less from the interference of the unselected optical carriers mixed with their own SCM channels, $i_{S C M}$, and the shot noise is determined mainly by the gain $G$ as shown in eqns. 21 and 24 , therefore the CNR value is not affected much by the wavelength number, $N_{W}$

\section{Comparison}

4.1 CSCM system using local oscillator, IM/DD SCM system using semiconductor laser amplifier and SCM system using Brillouin amplifier

Here we consider three different systems:

(a) the CSCM system (with the single-octave frequency allocation) using phase modulation (PM) and a local oscillator (LO) with power $P_{L O}[1]$

(b) the IM/DD SCM system using a travelling-wave semiconductor laser amplifier (SLA) [19,20]

(c) our amplitude modulated (AM) SCM system using a Brillouin amplifier with pump power $P_{p u m p}$.

We denote the CNRs of these three systems as $C N R_{L O_{C S C M}}, C N R_{S L A S C M}$, and $C N R_{F B A S C M}$, respectively. The improvement factors in receiver sensitivity [20] defined as the ratio of the receiver sensitivity, $P_{E}$, with the use of electronic preamplifier only over the receiver sensitivity, $P_{S}$, in the presence of local oscillator, the travelling-wave preamplifier and Brillouin amplifier are represented by $\gamma_{L O_{C S C Y}}, \gamma_{S L A S C M}$, and $\gamma_{F B A S C M}$, respectively. They can be expressed as (see Appendix 7),

$$
\begin{gathered}
C N R_{L O_{C S C M}} \\
=\frac{0.5 R^{2} P_{L O} P_{S} \beta^{2}}{\operatorname{var}\left(i_{t h}\right)+2 e R B P_{L O}+h_{3} K_{3} R^{2} P_{L O} P_{S} \beta^{6} / 32} \\
\gamma_{L O_{C S C M}}=\frac{R P_{L O}\left\{2 e B+\sqrt{ }\left[4 e^{2} B^{2}+2 \beta^{2} \operatorname{var}\left(i_{t h}\right) Z\right]\right\}}{2\left[\operatorname{var}\left(i_{t h}\right)+2 e R B P_{L O}\right]}
\end{gathered}
$$

where $Z \equiv \mathrm{CNR}^{-1}-h_{3} K_{3} \beta^{4} / 16$ in eqn. $28 . \beta$ is the $\mathrm{PM}$ index, $h_{3}$ takes into account the spectral form of intermodulation distortion and the associated filtering and $K_{3}$ is the number of third-order intermodulation distortion as given in Reference 1. Also,

$$
\begin{aligned}
& C N R_{S L A S C M} \\
& =\frac{\left(\eta_{i} \eta_{o} G P_{S} m R\right)^{2} / 2}{\left.\operatorname{var}\left(i_{t h}\right)+\left[\eta_{i} G P_{S}\left(\eta_{o} \eta_{e}\right)^{2} \sigma_{s i g-s P}^{2}+\left(\eta_{o} \eta_{e}\right)^{2} \sigma_{s p-s p}^{2}\right)\right] B} \\
& \gamma_{S L A_{S C M}}=\eta_{i} \eta_{o} G\left[\left(a^{2}+1\right)^{1 / 2}-a\right] \\
& a=\eta_{o} \eta_{e}^{2} \sigma_{s i g-s p}^{2} P_{E} /\left(2 \frac{\operatorname{var}\left(i_{t h}\right)}{B}\right) \\
& P_{E}=\frac{\left[2 \operatorname{var}\left(i_{t h}\right) C N R\right]^{1 / 2}}{R m} \\
& C N R_{F B A_{S C M}} \\
& =\frac{R^{2} m^{2} P_{S}^{2} G}{R^{2} m^{2} P_{S} P_{s p} / 2+e R B P_{S}\left(G+m^{2} N_{C} / 2\right)+2 N F k T B / r}
\end{aligned}
$$

$$
\gamma_{F B A S C M}=\frac{m\left[8 \operatorname{var}\left(i_{t h}\right)\right]^{1 / 2} G}{C N R^{1 / 2}\left[U+\sqrt{\left.\left(U^{2}+8 \frac{m^{2} G}{C N R} N F k T B / r\right)\right]}\right.}
$$

where $U \equiv\left(R m^{2} P_{s p} / 2\right)+e B\left(G+m^{2} N_{C} / 2\right)$ in eqn. 32. $\eta_{i}$, $\eta_{o}$ and $\eta_{e}$ in eqn. 29 are the input coupling efficiency of the amplifier, the total optical loss between amplifier and photodiode, and the photodiode quantum efficiency $\left(\eta_{e}=R h v / e\right)$, respectively. $P_{S}$ in eqns. 27,29 and 31 represent the received signal power of the CSCM system using LO, the total incident power on the SLA in the IM/DD SCM system, and the received signal power of the SCM system using FBA without amplification. $P_{E}$ in eqn. $30 b$ is the receiver sensitivity for an IM/DD SCM system using only electronic preamplifier. $m$ is the channel AM index (assumed equal for all channels). The third-order IMPs in eqn. 29 are $15-20 \mathrm{~dB}$ below the thermal noise and thus are negligible [21]. The other noise terms in eqn. 29 can be expressed as

$$
\begin{aligned}
& \sigma_{s i g-s p}^{2}=R^{2} F(G-1) \\
& \sigma_{s p / s p}^{2}=R^{2}(G-1)^{2} n_{s p} m_{t} \Delta v
\end{aligned}
$$

From Reference 22 we can find that for a semiconductor optical amplifier with gain $G$ of $20 \mathrm{~dB}$, noise figure $F$ of $5 \mathrm{~dB}$, population inversion factor $n_{\mathrm{SD}}$ of 2.2 , number of transverse modes $m_{t}$ of 2 , optical bandwidth $\Delta v$ of about $50 \mathrm{~nm}$, and fibre-to-amplifier coupling loss of $3 \mathrm{~dB}$ can provide a net fibre-to-fibre gain of $12 \mathrm{~dB}$. Then $\sigma_{\text {sig-sp }}^{2}$ is $1.2 \times 10^{-19} A^{2} / \mathrm{Hz} / \mathrm{mW}$ and $\sigma_{s p-s p}^{2}$ is $1.6 \times 10^{-20} A^{2} / \mathrm{Hz}$.

If we assume that all three systems have equal modulation index per channel $\beta=m=0.1$ and $N_{C}=20$, we may compare their CNR against $P_{S}$ for $P_{L O}=P_{\text {pump }}=0$, $1.5 \mathrm{dBm}, G=22,32 \mathrm{~dB}$ as shown in Figs. $8 a$ and $b$.
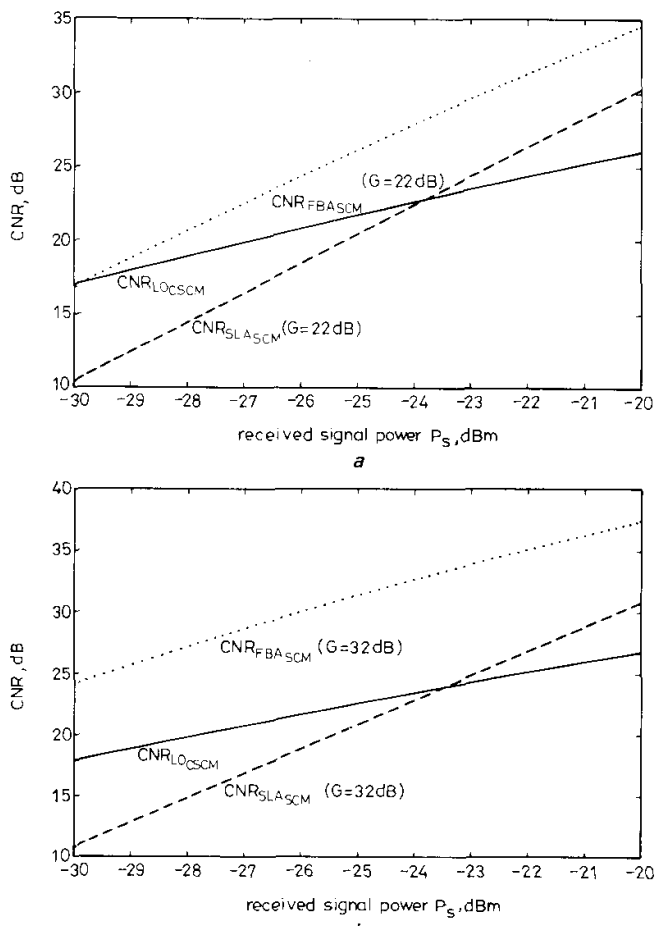

Fig. 8 CNR of the CSCM system using LO, of the IM/DD SCM system using $S L A$, and of the SCM using FBA against received signal power $P_{S}$ for $N_{C}=20, \beta=m=0.1$

a $P_{L O}=P_{\text {pump }}=0$ (corresponding to $G=22 \mathrm{~dB}$ )

$B=100 \mathrm{MHz}, \eta_{i}=\eta_{o}=0.5$

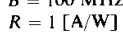

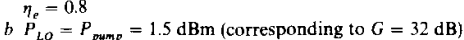


The CNR of the CSCM system is better in the lower signal power region; the CNR of the IM/DD system with SLA is better in the higher signal power region. However, the CNR of our SCM system with FBA performs better than the former two cases. Increasing the pump power from $0 \mathrm{dBm}$ to $1.5 \mathrm{dBm}$, we see that the CNR of our SCM system using FBA increases by about $7 \mathrm{~dB}$, but the CNRs of the CSCM system using LO and the SCM system using SLA remain unchanged (that is, in the saturation region) as shown in Figs. $8 a$ and $b$, respectively. To see the improvement of receiver sensitivity between the CSCM system with LO and the SCM system with FBA, we may compare $\gamma_{L O_{C S C M}}$ and $\gamma_{F B A S C M}$ under the same $P_{L O}$ and $P_{\text {pump }}$ for $\mathrm{CNR}=16 \mathrm{~dB}, 18 \mathrm{~dB}$, respectively, as shown in Fig. 9. The sensitivity improvement of the SCM system using FBA is superior to that of the CSCM system using LO at higher pump or LO power; the improvement increases as the CNR decreases. Also, the Brillouin approach is intrinsically simple and polarisation independent, whereas a coherent system is highly complex, polarisation dependent and costs significantly more. We compare the improvement between the SCM system with FBA and the IM/DD SCM system with SLA against the same gain $G$ for $C N R=16 \mathrm{~dB}, 18 \mathrm{~dB}$, respectively, as shown in Fig. 10. The receiver improvement of the SCM system using FBA is better in the lower gain region; but in the higher gain region that of the IM/DD SCM system using SLA is better. In general, for all amplifier gains of interest, a system that uses a semiconductor amplifier is better than the Brillouin approach. On the other hand, the polarisation dependence of semiconductor amplifiers, the difficulties of optical coupling, and stability have to be considered in practical implementation.

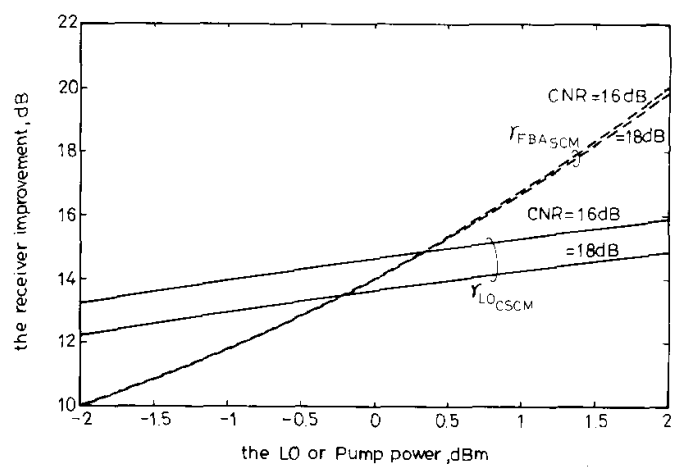

Fig. 9 Improvement of receiver sensitivity of CSCM system using LO, and the SCM system using FBA, respectively, under the same $P_{L O}$ and $P_{\text {pump }}$

$\mathrm{CNR}=16 \mathrm{~dB}, 18 \mathrm{~dB}$, respectively, for $N_{C}=20, \beta=m=0.1$

$B=100 \mathrm{MHz}$
4.2 IMIDD WDM-SCM system using optical preamplifier and WDM-CSCM system using FBA If we consider an IM/DD WDM-SCM system $[3,4]$ as shown in Fig. 11 which employs a travelling-wave SLA

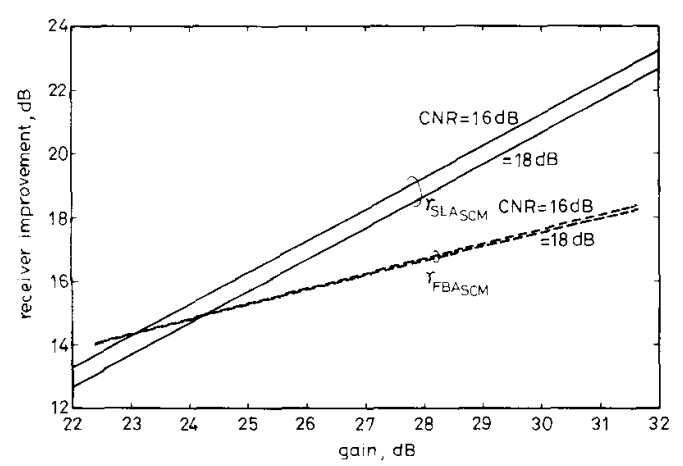

Fig. 10 Improvement of receiver sensitivity of the SCM system using $F B A$ and IM/DD SCM system using SLA under the same gain $G$ with $C N R=16 \mathrm{~dB}, 18 \mathrm{~dB}$, respectively

$N_{\mathrm{C}}=20$

$M=0.1=0.5$

$\eta_{i}=\eta_{0}=0.5$
$R=i[\mathrm{~A} / \mathrm{W}]$

$\begin{array}{ll}R= & =1 \mathrm{~A} / \mathrm{W} \\ \eta & =0.8\end{array}$

$\eta_{e}=0.8$
$B=100 \mathrm{MHz}$

as the preamplifier $[19,20]$ and uses the improved integrated-optic acousto-optic tunable filter (IAOTF) with total single-mode fibre-to-fibre insertion loss, $\eta_{f}[23$, 24], to select the desired wavelength in the WDM, then the CNR of such a system can be expressed as

$$
\begin{aligned}
& C N R_{S L A W D M-S C M} \\
& =\frac{\left(\eta_{f} \eta_{i} \eta_{o} G P_{S} m R\right)^{2} / 2}{\left.\operatorname{var}\left(i_{t h}\right)+\left[\eta_{f} \eta_{i} G P_{S}\left(\eta_{o} \eta_{e}\right)^{2} \sigma_{s i g-s p}^{2}+\left(\eta_{o} \eta_{e}\right)^{2} \sigma_{s p-s p}^{2}\right)\right] B}
\end{aligned}
$$

From eqns. 26 and 35 , we can obtain the CNR of WDM-SCM system using Brillouin amplifier and that of the IM/DD WDM-SCM system using IAOTF with SLA against received signal power $P_{S}$ as shown in Fig. 12 for $\eta_{f}=8.7 \mathrm{~dB}$ [23], $\eta_{i}=\eta_{o}=0.5(3 \mathrm{~dB}$ loss $), R=1(\mathrm{~A} / \mathrm{W})$, $\eta_{e}=0.8, m=0.1 B=100 \mathrm{MHz}, G=22 \mathrm{~dB}$ and $32 \mathrm{~dB}$, respectively. For gain varying from $32 \mathrm{~dB}$ to $22 \mathrm{~dB}$, the CNR of the WDM-SCM system using FBA is improved by $10 \mathrm{~dB}$, that of the WDM-SCM system using SLA is improved by $4 \mathrm{~dB}$. For the received power of $-25 \mathrm{dBm}$, the CNR of the WDM-SCM system using FBA is better than that of the WDM-SCM using SLA by more than $10 \mathrm{~dB}$; for the received power of $-15 \mathrm{dBm}$, the CNR of the WDM-SCM using FBA with $G=32 \mathrm{~dB}$ is still better than that of the WDM-SCM using SLA. In the WDM-SCM using SLA, there is an IAOTF which induces a high insertion loss $(8.7 \mathrm{~dB})$ and this causes its

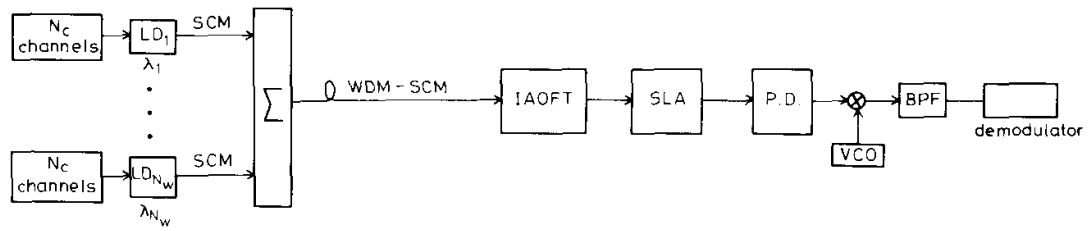

Fig. 11 System block diagram of IM/DD WDM-SCM system employing IAOTE and SLA

IEE PROCEEDINGS-J, Vol. 139, No.4, AUGUST 1992 
performance to be inferior to that of the WDM-SCM using FBA.

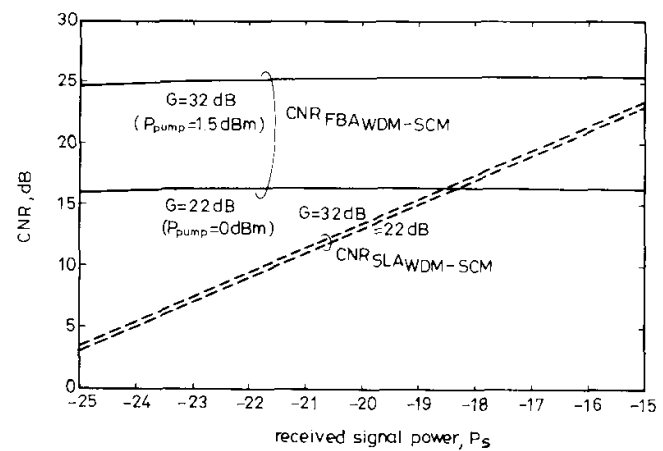

Fig. $12 C N R$ of the WDM-SCM system using FBA and the IM/DD $W D M-S C M$ system using IAOTF and SLA against received signal power $P_{S}$

$\eta_{f}=8.7 \mathrm{~dB}$

$\eta_{i}=\eta_{0}=0.5(3 \mathrm{~dB}$ loss $)$

$\eta_{i}=\eta_{0}=0.5(3 \mathrm{~dB}$ loss $)$
$G=22 \mathrm{~dB}$ and $32 \mathrm{~dB}$, respectively

$R=1[\mathrm{~A} / \mathrm{W}]$

$\eta_{e}=0.8$

and $B=100 \mathrm{MHz}$

\section{Conclusion}

A WDM-SCM system employing optical SBS narrowband amplification to select the desired channel is proposed in this paper. This system has the benefits of eliminating the need for polarisation control, cancelling the phase noise, and enhancing optical receiver sensitivities by amplification of the optical carrier.

At the receiver, we use a tunable pump laser to select the optical carrier in WDM by FBA and obtain and heterodyne IF signal without its being degraded by the phase noise. With appropriate received power and total optical modulation depth, the required CNR is achievable. The CNR is not influenced seriously by the number of optical carriers because the value of Brillouin gain is large and the beat noise produced by other unselected optical carriers with their SCM channels is not amplified.

We believe that such a structure may be attractive for the SCM system in which residual optical carrier power is stronger than its channels. Here we not only make use of this optical carrier but also achieve heterodyne detection without degradation of the phase noise.

We also see that our SCM using FBA outperforms the CSCM using LO and the SCM using SLA. As for the sensitivity improvement, the SCM using FBA is better than the CSCM using LO at the same pump and LO level in the high power range. The receiver improvement of the SCM using FBA is superior to that of the SCM using LO in the lower gain region. The WDM-SCM system using FBA does not need a wavelength filter, therefore such a structure may also be superior to the WDM-SCM system using SLA which has a high insertion loss wavelength filter for wavelength selection.

\section{References}

1 GROSS, R., and OLSHANSKY, R.: 'Multichannel coherent FSK experiments using subcarrier multiplexing techniques', $J$, Lightwave Technol., 1990, 8, (3), pp. 406-415

2 WAY, W.I.: 'Subcarrier multiplexed lightwave system design considerations for subscriber loop application', J. Lightwave Technol, $1989,7,(11)$, pp. 1806-1818

278
3 WESTLAKE, H.J., HILL, G.R., WICKENS, G.E., and CAVANAGH, B.P.: 'Subcarrier multiplexed transmission using wavelength division multiplexing and optical amplifier', Electron. Lett., $1989,25,(10)$, pp. $632-634$

4 LIEW, S.C., and CHEUNG, K.W.: "A broad-band optical network based on hierarchical multiplexing of wavelengths and RF subbased on hierarchical multiplexing of wavelengths and
carriers', J. Lightwave Technol., 1989, 7, (11), pp. 1825-1835

5 CHRAPLYVY, A.R., and TKACH, R.W.: 'Narrow tunable optical filter for channel selection in densely packed WDM systems', Electron. Lett., 1986, 22, pp. 1084-1085

6 TKACH, R.W., CHRAPLYVY, A.R., and DEROSIER, R.M.: 'Performance of a WDM network based on stimulated Brillouin scattering', IEEE Photonics Technol. Lett., 1989, (5), pp. 111-112

7 GROSS, R., OLSHANSKY, R., and SCHMIDT, M.: 'Coherent FM-SCM system using DFB lasers and a phase noise cancellation circuit', IEEE Photonics Technol. Lett., 1990, 2, (1), pp. 66-68

8 ATKINS, C.G., COTTER, D., SMITH, D.W., and WYATT, R.: Application of Brillouin amplification in coherent optical transmission', Electron. Lett., 1986, 22, pp. 556-558

9 OLSSON, N.A., and VANDER ZEIL, J.P.: 'Characteristics of a semiconductor laser pumped Brillouin amplifier with electronically controlled bandwidth', J. Lightwave Technol., 1987, LT-5, pp. $147-153$

10 COTTER, D.: 'Stimulated Brillouin scattering in monomode optical fiber', J. Opt. Commun., 1983, 4, pp. 10-19

11 ARNAUD, J.A.: 'Enhancement of optical receiver sensitivities by amplification of the carrier', IEEE J. Quantum Electron., 1968 , QE-4, (11), pp. 893-899

12 MOCHIZUKI, K., EDAGAWA, N., and IWAMOTO, Y.: 'Amplified spontaneous Raman scattering in fiber Raman amplifier', $J$ Lightwave Technol., 1986, LT-4, pp. 1328-1333

13 CHILDS, R.B., and O'BYRNE, V.A.: 'Multichannel AM video transmission using a highpower Nd:YAG laser and linearized external modulator', J. Sel. Area Commun., 1990, 8, (7), pp. 1369-1376

14 BODEEP, G.E., TARCIE, T.E.: 'Comparison of second and third order distortion in intensity modulated InGaAsP lasers and on $\mathrm{LiNbO}_{3}$ external modulator'. Tech. Dig. Opt. Fiber Conf. (Houston TX), Feb. 6-9, 1989

15 AGRAWAL, G.P.: 'Nonlinear fiber optics' (Academic Press. Inc., New York, 1989) chap. 9

16 SMITH, R.G.: 'Optical power handling capacity of low loss optical fibers as determined by stimulated Raman and Brillouin scattering', Appl. Opt., 1972, (11), pp. 2489-2494

17 ABUELMA'ATTI, M.T.: 'Carrier-to-intermodulation performance ABUELMA'ATTI, M.T.: 'Carrier-to-intermodulation performance
of multiple FM/FDM carriers through a GaAlAs heterojunction laser diode', IEEE Trans., 1985, COM-33, (3), pp. 246-248

18 OLSHANSKY, R., LANZISERA, V.A., and HILL, P.M.: 'Subcarrier multiplexed lightwave systems for broadband distribution', $J$. Lightwave Technol., 1989, LT-7, (9), pp. 1329-1341

19 JOYCE, G.R., LANZISERA, V., and OLSHANSKY, R.: 'Improved Sensitivity of 60 video channel FM-SCM receiver with semiconductor optical preamplifier', Electron. Lett., 1989, 25, pp. 499-501

20 OLSHANSKY, R.: 'Optical preamplifiers for subcarrier multiplexed OLSHANSKY, R.: 'Optical preamplifiers for subcarrier mult
lightwave systems', Electron. Lett., 1987, 23, pp. 1196-1 197

21 OLSHANSKY, R., and LANZISERA, V.A.: ' 60 channel FM video subcarrier multiplexed optical communications system', Electron. Lett., 1987, 23, pp. 1196-1197

22 MUKAI, T., and SAITOH, T.: $5.2 \mathrm{~dB}$ noise figure in a $1.5 \mu \mathrm{m}$ InGaAsP travelling-wave laser amplifier', Electron. Lett., 1987, 24, pp. $216-218$

23 CHEUNG, K.W., LIEW, S.C., and LO, C.N.: 'Simultaneous fivewavelength filtering at $2.2 \mathrm{~nm}$ wavelength separation using integrated-optic tunable filer with subcarrier detection', Electron integrated-optic tunable filter with
Lett., $1989,25,(25)$, pp. $636-637$

24 HEFFNER, B.L., BARAN, D.A., YI-YAN, A., and CHEUNG, K.W: :Improved acoustically-tunable optical filter on $x$-cut $\mathrm{LiNbO}_{3}{ }^{\prime}$, Electron. Lett., 1989, 24, pp. 1562-1563

\section{Appendix}

The CNR and receiver sensitivity $P_{E}$ of a CSCM system which uses only an electronic preamplifier can be expressed as

$$
\begin{aligned}
& C N R=\frac{0.5 R^{2} P_{E}^{2} \beta^{2}}{\operatorname{var}\left(i_{t h}\right)+2 e R B P_{E}+R^{2} h_{3} K_{3} P_{E}^{2} \beta^{6} / 32} \\
& P_{E}=\frac{2 e B+\sqrt{\left[4 e^{2} B^{2}+2 \beta^{2} \operatorname{var}\left(i_{t h}\right) Z\right]}}{R \beta^{2} Z}
\end{aligned}
$$

IEE PROCEEDINGS-J, Vol. 139, No. 4, AUGUST 1992 
where $Z \equiv C N R^{-1}-h_{3} K_{3} \beta^{4} / 16$. From eqn. 27, we can obtain the receiver sensitivity $P_{S}$ and the improvement of the receiver sensitivity for the system using a local oscillator, respectively, as

$$
\begin{aligned}
P_{S}= & \frac{\operatorname{var}\left(i_{t h}\right)+2 e R B P_{L O}}{0.5 R^{2} \beta^{2} P_{L O} Z} \\
\gamma_{L O_{C S C M}}= & \frac{P_{E}[\text { in eqn. 37] }}{P_{S} \text { [in eqn. 38] }} \\
& =\frac{R P_{L O}\left[2 e B+\sqrt{ }\left(4 e^{2} B^{2}+2 \beta^{2} i_{t h}^{2} Z\right)\right]}{2\left[\operatorname{var}\left(i_{t h}\right)+2 e R B P_{L o}\right]}
\end{aligned}
$$

From eqn. 31, we may express the receiver sensitivity $P_{S}$ for transmission of only one wavelength (that is, $N_{W}=1$ in eqn. 26) and $m_{o}^{2} / N_{C}=m^{2}$ as

$$
\left(\frac{R^{2} m^{2} G}{C N R}\right) P_{S}^{2}-R U P_{S}-2 N F k T B / r=0
$$

where $U \equiv\left(R m^{2} P_{s p} / 2\right)+e B\left(G+m^{2} N_{c} / 2\right)$. Then we can solve eqn. 4 to obtain $P_{S}$ as,

$$
P_{S}=\frac{U+\sqrt{[}\left[U^{2}+8\left(m^{2} G / C N R\right) N F k T B / r\right]}{2\left(R m^{2} G / C N R\right)}
$$

and the improvement in the receiver sensitivity using FBA can be expressed as

$$
\begin{aligned}
\gamma_{F B A S C M} & \\
= & \frac{P_{E}[\text { in eqn. } 30 b]}{P_{S}[\text { in eqn. 41] }} \\
= & \frac{m\left[8 \operatorname{var}\left(i_{t h}\right)\right]^{1 / 2} G}{C N R^{1 / 2}\left\{U+\sqrt{\left.\left[U^{2}+8\left(m^{2} G / C N R\right) N F k T B / r\right]\right\}}\right.}
\end{aligned}
$$

